\title{
IL-4, IL-6, IL-10, IL-17A and vascular endothelial growth factor in the vitreous of patients with proliferative diabetic retinopathy
}

\author{
Valery Chernykh $^{1}$, Evgeny Smirnov ${ }^{1}$, Yegor Varvarinsky ${ }^{1}$, Dmitry Chernykh ${ }^{1}$, Olga Obukhova ${ }^{2}$, \\ Aleksandr Trunov ${ }^{1,2}$ \\ ${ }^{1}$ Novosibirsk Branch Federal State Institution “Intersectoral Research and Technology Complex” “Eye microsurgery”, Novosibirsk, \\ Russia \\ ${ }^{2}$ Research Center of Clinical and Experimental Medical, Siberian Branch of Russian Academy of Medical Sciences, Novosibirsk, \\ Russia \\ Email: Trunov1963@yandex.ru
}

Received 8 December 2013; revised 9 January 2014; accepted 22 January 2014

Copyright (C 2014 Valery Chernykh et al. This is an open access article distributed under the Creative Commons Attribution License, which permits unrestricted use, distribution, and reproduction in any medium, provided the original work is properly cited. In accordance of the Creative Commons Attribution License all Copyrights (C) 2014 are reserved for SCIRP and the owner of the intellectual property Valery Chernykh et al. All Copyright (C) 2014 are guarded by law and by SCIRP as a guardian.

\section{ABSTRACT}

In current article, results of the determination of the concentrations of vascular endothelial growth factor (VEGF), and some interleukins (IL-4, IL-6, IL-10, IL-17A) in the vitreous of patients with proliferative diabetic retinopathy are presented. We have found that in the mechanism of development of diabetic retinopathy, significant role plays an activation of local inflammation process and vascular proliferation. This is evidenced by a significant increase in the vitreous concentration of IL-4, IL-6, IL-10, IL-17A and vascular endothelial growth factor. Identified correlations between VEGF and IL-17A, between VEGF and IL-4, and between IL-17A and IL-4 show the relationship of inflammation and proliferation processes in the pathogenesis of diabetic retinopathy.

\section{KEYWORDS} Diabetic Retinopathy; Cytokines; Vascular
Endothelial Growth Factor

\section{INTRODUCTION}

In today's world, there are 200 million people, suffering from diabetes mellitus, and their numbers every 10 - 15 years increased almost twice. Ophthalmic complications, which arise during the development of diabetes mellitus, are the crucial medical and social problems. The urgency of this problem is associated with significant role of ophthalmic complications of diabetes mellitus in the structure of visual impairment and irreversible blindness. These severe complications lead to disability of patients with diabetes mellitus and are a serious social consequences for the individual and for society as a whole [1-3].

Despite the progress of modern medicine in the implementation of methods of prevention and treatment of diabetic lesions of the organ of vision, one of the worst specific defects of eye in diabetes mellitus is diabetic retinopathy. It is known that the prevalence of retinopathy among patients with diabetes mellitus may reach $80 \%-90 \%$ and depends on many factors. These include the duration of diabetes mellitus, completeness of conducted comprehensive treatment, the effectiveness of the control of glucose level in blood serum and a number of other factors. The progression of diabetic retinopathy may be complicated by recurrent intraocular hemorrhage, retinal and vitreous fibrosis, traction retinal detachment, optic atrophy, which leads to the development of irreversible blindness [1,4-6].

At present, the pathogenesis of diabetes mellitus is seen as a multifactorial process. Its pathogenetic basis is local and systemic vascular and metabolic disorders that lead to the appearance of lesions of the vision.

The scientific literature shows that patients with diabetic retinopathy revealed activation of inflammatory reactions, violations of the functional state of the immune system and also marked imbalance processes of intercellular interrelationships inductors-cytokines, matrix metalloproteinases, growth factors and other elements. Identified disorders indicate their absolute impor- 
tance in the pathogenesis of diabetic retinopathy [7-11]. In recent years, great importance is attached to the vascular endothelial growth factor in the mechanisms of development of proliferative diabetic retinopathy [1215].

However, many of the mechanisms of the pathogenesis of proliferative diabetic retinopathy remain unclear. Questions remain about the role of pro-inflammatory, anti-inflammatory and regulatory cytokines in diabetic retinopathy. The relationship of cytokines with the factors activating proliferation, also requires further study $[4,10]$.

The aim of the present work was to study the content of interleukins IL-4, IL-6, IL-10, IL-17A and vascular endothelial growth factor (VEGF) in the vitreous of patients with proliferative diabetic retinopathy.

\section{MATERIALS AND METHODS}

According to the purpose of study for the period from year 2011 to 2012, we examined 38 patients who underwent surgical treatment for traction retinal detachment. The patients' ages ranged from 25 to 78 years (mean age -52.6 years). In the study group were 20 women and 18 men.

All patients gave informed consent to participate in the study and for the operation and fence of the vitreous, and the use of research data for scientific purposes, which is consistent with the ethical standards developed in accordance with the Declaration of Helsinki.

The criterion for inclusion in the study was the presence of patients traction retinal detachment, about which was conducted a surgical treatment.

Inclusion criteria for the main group of patients was the availability of diabetes and proliferative diabetic retinopathy.

Inclusion criterion for a comparison group of was the absence in patients of diabetes and diabetic proliferative retinopathy.

Exclusion criteria for both groups was availability of the acute exacerbation of chronic inflammatory diseases of eye, primary open-angle glaucoma, uveitis of various etiologies, as well as autoimmune and neoplastic processes of any location.

Thus formed the core group (I group) -22 patients with traction retinal detachment against the background of proliferative diabetic retinopathy. The average age of patients in the group was 50.5 years (range, 46 - 62 years). The number of women in the group was 14 , men -8 . Diabetes mellitus Type 1 was in 5 patients. Diabetes mellitus Type 2-in 17 patients. The mean hemoglobin A1c level was 9.9\% (range, 6.9\% - 12.2\%), and the mean duration of diabetes was 12.4 years (range, 8 - 16 years).

The diagnosis proliferative diabetic retinopathy is on the basis of the survey, including: visometry, delimitation of the field of view and the presence of scotomas, binocular ophthalmoscopy with the use of forehead ophthalmoscope Heine Omega 200 and 20 diopter lens, slit lamp Karl Zeiss SL 115 Classic and lenses Ocular Max Field 78 diopters dimensional ultrasound scan setting Tomey UD 1000.

The comparison group (II group) was 16 patients with traction retinal detachment, without a history of diabetes mellitus and had no signs of diabetic retinopathy. Mean age of patients in group was $55.5 \pm 2.6$ years. In the comparison group were 6 women and 10 men.

Surgical treatment of retinal detachment in patients of both groups was carried out by a standard procedure back WAY vitrectomy 25G, 23G using operating systems "Constellation Vision System" (Alcon, USA), "Stellaris PC” (Bausch + Lomb, USA), Assistant (Optikon, Italy). As the investigated material was used vitreous picked in the initial stages of vitrectomy. For the exception of hit of salt-free solution (BSS), fence of material was carried with the air tamponade. Further mobilization of retina, retinal fixation with perfluororganic compounds (PFOS), endolazerkoagulation.

Biological material obtained was placed into tubes and centrifuged for 10 minutes at $1500 \mathrm{rpm}$, the resulting supernatant was placed in plastic tubes, frozen at $70^{\circ} \mathrm{C}$ and, subsequently, used for the laboratory diagnosis. Samples for analysis were used within 6 months after collecting.

Determination of IL-4, IL-6, IL-10 and IL-17A in the vitreous was carried out using commercial test systems for immunoassay produced by “Cytokine” company (Russia) at the manufacturer's instructions.

Determination of vascular endothelial growth factor (VEGF) in the vitreous was performed by ELISA on commercial test systems, produced by "Vector-BEST" (Novosibirsk, Russia) at the manufacturer's instructions.

ELISA results were recorded on a vertical photometer “Uniplan” at a wavelength of $450 \mathrm{~nm}$.

The resulting digital data were subjected to statistical analysis and presented in tabular form. Data analysis was performed using the software package Statistics 10 be StatSoft Inc. The significance of the differences in the variation series of unrelated samples was evaluated using the Mann-Whitney test. Data in tables are presented as $\mathrm{M} \pm \mathrm{m}$, where $\mathrm{M}-$ mean, $\mathrm{m}$ - error of the mean. Considered significant difference between the compared series with a confidence level of probability 95\% ( $p<0.05)$. Correlation analysis was carried out using Spearman rank correlation.

\section{RESULTS AND DISCUSSION}

The studies obtained the results shown in Table 1 . 
Table 1. Contents VEGF, IL-4, IL-6, IL-10, IL-17A in the vitreous of patients with traction retinal detachment and diabetic retinopathy (Group I) and with no signs of retinopathy (Group II) (M \pm m).

\begin{tabular}{ccc}
\hline Index Groups & $\begin{array}{c}\text { Group I Patients with } \\
\text { diabetic retinopathy }(\mathrm{n}=22)\end{array}$ & $\begin{array}{c}\text { Group II A comparison } \\
\text { group }(\mathrm{n}=16)\end{array}$ \\
\hline VEGF, pg/ml & $1491.0 \pm 183.1^{*}$ & $65.2 \pm 11.2$ \\
IL-4, pg/ml & $32.5 \pm 9.4^{*}$ & $8.1 \pm 0.5$ \\
IL-6, pg/ml & $64.2 \pm 10.4^{*}$ & $32.8 \pm 8.7$ \\
IL-10, pg/ml & $4.43 \pm 0.4$ & $4.59 \pm 0.6$ \\
IL-17A, pg/ml & $184.2 \pm 18.7^{*}$ & $64.3 \pm 4.8$ \\
\hline
\end{tabular}

*difference on the value of the corresponding figure for the comparison group was significant at $\mathrm{p}<0.05$.

Vascular endothelial growth factor (VEGF)—biologically active substance capable of binding and activation by membrane receptors (receptor-1 VEGF and receptor-2 VEGF), a signaling cascade run. The result of this stage is the stimulation of growth and proliferation of vascular endothelial cells. After the formation of new blood vessels, VEGF plays the role of survival factor by inhibition of apoptosis of endothelial cells. Concentration of vascular endothelial growth factor in the vitreous of patients with proliferative diabetic retinopathy was significantly (by more than 23 times) higher, than the index of patients with traction retinal detachment without signs of proliferative diabetic retinopathy $(p<0.001)[13,16,17]$.

Interleukin-17A is a pro-inflammatory cytokine that is secreted primarily by activated T-lymphocytes. One of the functions of IL-17A is the stimulation of synthesis of pro-inflammatory cytokines TNF- $\alpha$, IL- $1 \beta$, as well as cell adhesion molecules and other factors $[18,19]$. The concentration of pro-inflammatory cytokines IL-17A in the vitreous of patients with proliferative diabetic retinopathy was significantly (2.8 times) higher than the index of patients with traction retinal detachment without signs of proliferative diabetic retinopathy $(\mathrm{p}<0.001)$.

IL-4-cytokine has anti-inflammatory properties. It is an activator of T-helper response of Type 2, which defines the importance of IL-4 in the development of the immune response during inflammatory and autoimmune processes. The concentration of IL-4 in the vitreous of patients with proliferative diabetic retinopathy was significantly (4 times) higher than the index of patients with traction retinal detachment without signs of proliferative diabetic retinopathy $(\mathrm{p}<0.001)$.

IL-6-proinflammatory cytokine plays an important role in the mechanisms of chronization of inflammatory processes, including autoimmune etiology [20]. IL-6 concentration in the vitreous of patients with traction retinal detachment and proliferative diabetic retinopathy was significantly (by 1.9 times) higher than the index of patients without signs of proliferative diabetic retinopathy $(\mathrm{p}<0.001)$.

IL-10 - anti-inflammatory cytokine involved in the processes of chronization and has immunosuppressive properties. In addition, IL-10 is involved in the regulation of the synthesis of VEGF. When comparing the content of IL-10 in patients of examined groups, the accuracy of the values of the differences was not found. The data obtained are different from those in the scientific literature that suggests increasing the content of this cytokine in patients with proliferative diabetic retinopathy, which plays a significant role in its pathogenesis [21]. Probably the activity of its products may be associated with the timing of the pathological process that requires further study.

To evaluate the diagnostic and prognostic value of any parameter is important not only change its content in biological substances, but the evaluation of the severity and direction of the relationship between the studied parameters in the pathogenesis of the pathological process.

The study of the vitreous of patients of the main group was identified certain correlations, which presented in Table 2.

Of the identified interactions, reliability is the direct significant correlative relationship between the concentrations of VEGF and IL-17A and between the concentrations of VEGF and IL-4, as well as between the concentrations of IL-17A and IL-4. Identified correlations may be a sign of the relationship between processes of proliferation and inflammation in the pathogenesis of proliferative diabetic retinopathy.

Thus, our study has shown that in mechanisms of the development of proliferative diabetic retinopathy significant role played the high activity of local immune responses and inflammation process. This is evidenced by significantly higher levels of cytokines IL-17A, IL-6 and IL4 in the vitreous of patients with diabetic retinopathy compared with the control group.

Increased production of these cytokines apparently leads to the activation of cell proliferation of the vascular wall. Perhaps proof of this is more than 20-fold increase in the content of vascular endothelial growth factor in the vitreous. Identified correlations indicate the relationship

Table 2. Correlation relationship between the figures, determined in the vitreous of patients with diabetic retinopathy (Group I).

\begin{tabular}{cccc}
\hline Index 1 & Index 2 & $\begin{array}{c}\text { The correlation } \\
\text { coefficient (R) }\end{array}$ & $\begin{array}{c}\text { Significant } \\
\text { difference (p) }\end{array}$ \\
\hline VEGF & IL-17A & 0.51 & $<0.05$ \\
IL-4 & IL-17A & 0.43 & $<0.05$ \\
IL-4 & VEGF & 0.51 & $<0.05$ \\
\hline
\end{tabular}


of inflammatory and proliferative processes.

\section{REFERENCES}

[1] Balashevich, L.I. (2004) Ocular manifestations of diabetes. Medical Academy of Postgraduate Education, St. Petersburg, 382.

[2] Libman, E.S., and Shahova, E.V. (2006) Blindness and disability due to pathology of the vision in Russia. Journal of Ophthalmology, 122, 35-37.

[3] Bhavsar, A.R. (2006) Diabetic retinopathy: The latest in current management. Retina, 26, 71-79. http://dx.doi.org/10.1097/00006982-200607001-00016

[4] Lim, A., Stewart, J., Chui, T.Y., Lin, M., Ray, K., Lietman, T., Porco, T., Jung, L., Seiff, S. and Lin, S. (2008) Prevalence and risk factors of diabetic retinopathy in a multi-racial underserved population. Ophthalmic Epidemiology, 15, 402-409. http://dx.doi.org/10.1080/09286580802435179

[5] Kollias, A.N. and Ulbig, M.W. (2010) Diabetic retinopathy: Early diagnosis and effective treatment. Deutsches Ärzteblatt international, 107, 75-83.

[6] Sadaka, A. and Giuliari, G.P. (2012) Proliferative vitreoretinopathy: Current and emerging treatments. Journal of Clinical Ophthalmology, 6, 1325-1333.

[7] Chernykh, V.V., Lysikov, A.G., Obukhova, O.O., Gorbenko, O.M., Shvayuk, A.P. and Trunov, A.N. (2011) Features of local immunoinflammatory process at nonproliferative diabetic retinopathy. Bulletin of the Novosibirsk State University, 9, 164-168.

[8] Khodjaev, N.S., Chernykh, V.V., Romenskaya, I.V., Kuntysheva, K.E. and Trunov, A.N. (2011) Effect of laser coagulation retina on clinical-laboratory parameters in patients diabetic macular edema. Bulletin of the Novosibirsk State University, 9, 48-53.

[9] Kowluru, R.A., Zhong, Q. and Kanwar, M. (2010) Metabolic memory and diabetic retinopathy: Role of inflammatory mediators in retinal pericytes. Experimental Eye Research, 90, 617-623. http://dx.doi.org/10.1016/j.exer.2010.02.006

[10] Rasier, R., Gormus, U., Artunay, O., Yuzbasioglu, E., Oncel, M. and Bahcecioglu, H. (2010) Vitreous levels of VEGF, IL-8, and TNF-alpha in retinal detachment. Current Eye Research, 35, 505-509. http://dx.doi.org/10.3109/02713681003597248

[11] Symeonidis, C., Papakonstantinou, E., Androudi, S., Rotsos, T., Diza, E., Brazitikos, P., Karakiulakis, G. and Dimitrakos, S.A. (2011) Interleukin-6 and the matrix metalloproteinase response in the vitreous during proliferative vitreoretinopathy. Cytokine, 54, 212-217. http://dx.doi.org/10.1016/j.cyto.2011.02.001

[12] Slepova, O.S., Neroev, V.V., Ilyukhin, P.A. and Sarygina, O.I. (2012) Immunological control of in the surgical treatment of patients with proliferative diabetic retinopathy with preliminary introduction to the vitreous Lucentis. Russian Ophthalmological Journal, 1, 69-74.

[13] Citirik, M., Kabatas, E.U., Batman, C., Akin, K.O. and Kabatas, N. (2012) Vitreous vascular endothelial growth factor concentrations in proliferative diabetic retinopathy versus proliferative vitreoretinopathy. Ophthalmic Research, 47, 7-12. http://dx.doi.org/10.1159/000324200

[14] Pennock, S. and Kazlauskas, A. (2012) Vascular endothelial growth factor A competitively inhibits platelet-derived growth factor (PDGF)-dependent activation of PDGF receptor and subsequent signaling events and cellular responses. Molecular and Cellular Biology, 32, 1955-1966. http://dx.doi.org/10.1128/MCB.06668-11

[15] Murugeswari, P., Shukla, D., Rajendran, A., Kim, R., Namperumalsamy, P. and Muthukkaruppan, V. (2008) Proinflammatory cytokines and angiogenic and anti-angiogenic factors in vitreous of patients with proliferative diabetic retinopathy and eyes disease. Retina, 28, 817824. http://dx.doi.org/10.1097/IAE.0b013e31816576d5

[16] Mohan, N., Monickaraj, F., Balasubramanyam, M., Rema, M. and Mohan, V. (2012) Imbalanced levels of angiogenic and angiostatic factors in vitreous, plasma and postmortem retinal tissue of patients with proliferative diabetic retinopathy. Journal of Diabetes and its Complications, 26, 435-441. http://dx.doi.org/10.1016/j.jdiacomp.2012.05.005

[17] Wakabayashi, Y., Usui, Y., Okunuki, Y., Kezuka, T., Takeuchi, M., Goto, H. and Iwasaki, T. (2010) Correlation of vascular endothelial growth factor with chemokines in the vitreous in diabetic retinopathy. Retina, 30, 339-344. http://dx.doi.org/10.1097/IAE.0b013e3181bd2f44

[18] Kang, M.H., Kim, M.K., Lee, H.J., Lee, H.I., Wee, W.R. and Lee, J.H. (2011) Interleukin-17 in various ocular surface inflammatory diseases. Journal of Korean Medical Science, 26, 938-944.

http://dx.doi.org/10.3346/jkms.2011.26.7.938

[19] Regan, D.P., Aarnio, M.C., Davis, W.S., Carmichael, K.P., Vandenplas, M.L., Lauderdale, J.D. and Moore, P.A. (2012) Characterization of cytokines associated with Th17 cells in the eyes of horses with recurrent uveitis. Veterinary Ophthalmology, 15, 145-152. http://dx.doi.org/10.1111/j.1463-5224.2011.00951.x

[20] Neurath, M.F. and Finotto, S. (2011) IL-6 signaling in autoimmunity, chronic inflammation and inflammationassociated cancer. Cytokine \& Growth Factor Reviews, 22, 83-89. http://dx.doi.org/10.1016/j.cytogfr.2011.02.003

[21] Suzuki, Y., Nakazawa, M., Suzuki, K., Yamazaki, H. and Miyagawa, Y. (2011) Expression profiles of cytokines and chemokines in vitreous fluid in diabetic retinopathy and central retinal vein occlusion. Japanese Journal of Ophthalmology, 55, 256-263.

http://dx.doi.org/10.1007/s10384-011-0004-8 